\title{
Computation of Resonant Frequency of Gap-coupled Ring Microstrip Antennas
}

\author{
Pradeep Kumar \\ Department of Electronics and Communication Engineering, Jaypee University of Information Technology, Waknaghat-173234, India
}

\begin{abstract}
In this paper, an analytical model for computing the resonant frequency of the gap-coupled ring microstrip patch antennas is developed. The analytical model is based upon the cavity model along with circuit theory. Using the field expressions and boundary conditions, the transcendental equation for the structure is developed. The analytically computed results are compared with the simulated results. The simulation work is carried out by using computer simulation technology (CST) microwave studio simulator. The comparison between simulated and computed results shows good agreement.
\end{abstract}

Keywords: Ring microstrip antenna, gap-coupling, resonant frequency, analytic model, parasitic element.

\section{Introduction}

Microstrip patch antennas are preferred in many applications such as aircraft, missile and wireless communications due to their many attractive features like light weight, small size, low fabrication cost, easy implementation of feeding technique on same substrate, etc ${ }^{[1-4]}$. However, these antennas have limitations of low gain and narrow bandwidth. Many techniques have been used to enhance the bandwidth of microstrip antennas such as by changing the parameters of the substrate ${ }^{[5,6]}$, by loading ${ }^{[7-11]}$, using stack coupled microstrip antennas ${ }^{[12,13]}$, using gap-coupled microstrip antennas ${ }^{[14-19]}$, etc. Gap-coupling is one of the potential methods to increase the bandwidth of the antenna. In gap-coupling method, two microstrip patches are placed on the same substrate with some gap distance. One patch is fed by a suitable feeding technique and the other is fed by gap-coupling. This method is suitable for enhancing the bandwidth of the antenna and for the dual band microstrip antenna. The analytical models of the antennas are helpful for designing of the antenna with desired specifications.

In this paper, an analytical model for computation of resonant frequencies of gap-coupled circular ring microstrip patch antenna has been derived. Gap-coupling is one of the potential methods of increasing the bandwidth of the microstrip antenna as it generates two close resonances in the structure and by optimizing the dimensions, the two resonances can be merged for providing wider bandwidth ${ }^{[19]}$. The major advantages of ring antennas are the smaller size and wider bandwidth as compared to the circular and rectangular antenna ${ }^{[1]}$. So in this paper, the gap-coupled ring antenna structure is used as it is more capable to increase the bandwidth. Rest of the paper is organized as follows: The geometrical configuration of the gap-coupled circular microstrip ring antennas is presented in Section 2. The field expressions and analysis of the structure are presented

Regular paper

Manuscript received April 27, 2013; accepted September 30, 2013 in Section 3. Section 4 presents the simulated and analytically computed results and finally the Section 5 concludes the work.

\section{Antenna geometry}

The geometrical configuration of the gap-coupled circular microstrip ring antenna is depicted in Fig. 1. The inner and outer radii of inner ring are $a$ and $b$, respectively. The inner and outer radii of outer ring are $c$ and $d$, respectively. The outer ring is the feed ring and is excited by microstrip line feeding technique as shown in Fig. 1. The inner ring is the parasitic element and is excited by gap-coupling. The height and dielectric constants of the substrate are $h$ and $\epsilon_{r}$, respectively.
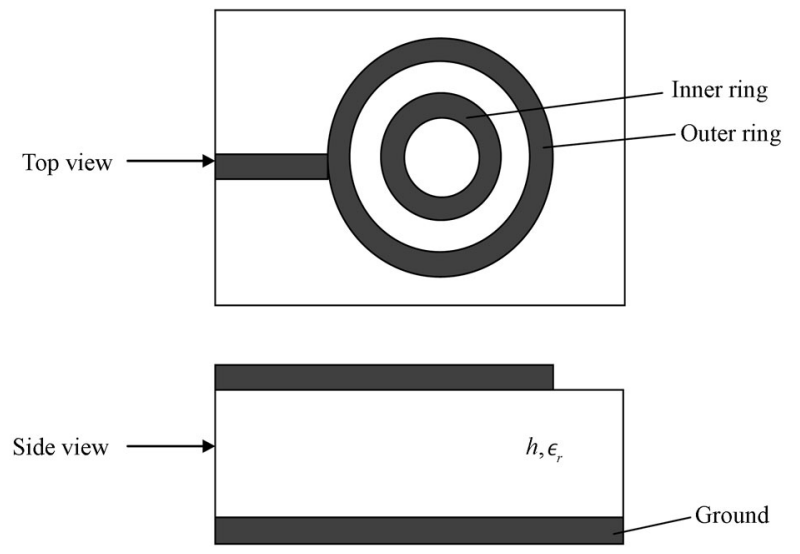

Fig. 1 Geometry of gap-coupled circular microstrip ring antenna

\section{$3 \quad$ Fields and analysis}

The analytical configuration of the gap-coupled circular ring microstrip antenna is shown in Fig. 2. For the analysis of the structure, the structure is divided into two regions 
as shown in Fig. 2. The inner and outer radii of the region I are $a$ and $b$, respectively; and the inner and the outer radii of region II are $c$ and $d$, respectively. The gap distance between two regions is denoted by $s$. Now, the field expressions for a given $T M_{n p}$ mode in these two regions are considered, where $n$ is the number of sign changes in the field along radial direction, and $p$ denotes the number of sign variations along the $z$-axis. Here, first two modes of the structure are computed for taking the potential advantage of this structure, i.e., for broad-banding only first two modes are used. However, the proposed model is also applicable for computation of higher modes.

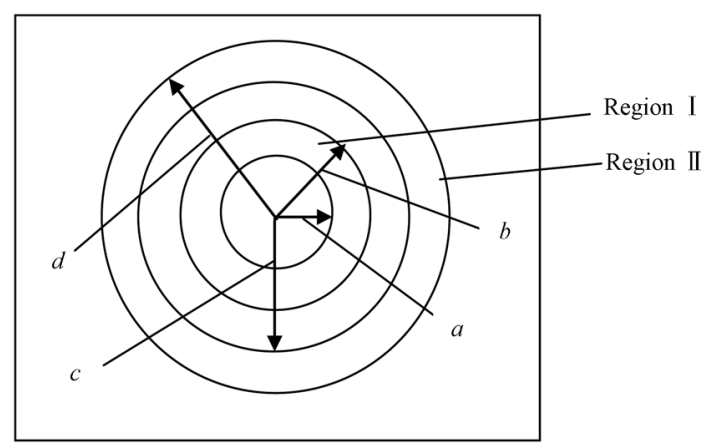

Fig. 2 Two regions of the structure

In region I, the wave equation for the electric field $E$ between ring and ground plane can be written as

$$
\left(\nabla^{2}+k^{2}\right) E=0
$$

where $k=\frac{2 \pi \sqrt{\epsilon_{r}}}{\lambda_{0}}$, and $\lambda_{0}$ is the free space wavelength. The above wave equation can be written in the cylindrical coordinate system as

$$
\frac{\partial\left(r \frac{\partial E}{\partial r}\right)}{r \partial r}+\frac{1}{r^{2}} \frac{\partial^{2} E}{\partial \phi^{2}}+k^{2} E=0
$$

where $r, \phi$, and $z$ are usual cylindrical coordinates. The electric field must satisfy the above wave equation and the magnetic wall boundary condition. The solution of the wave equation in this region is given as

$$
E_{z}^{1}=-\mathrm{j} \omega \mu\left[C_{1} J_{n}(k r)+C_{2} N_{n}(k r)\right] \cos (n \phi)
$$

where $E_{z}^{1}$ is the electric field in the $z$-direction for region I. $\omega, \mu$, and $k$ are the angular frequency, permeability, and propagation constant, respectively. $J_{n}(k r)$, and $N_{n}(k r)$ are the Bessel function of first kind and second kind of order, respectively. $C_{1}$, and $C_{2}$ are the amplitude constants. As electric field is having only $z$ component and is constant with respect to $z$. The relation between $E$-field and $H$-field is given by

$$
\begin{aligned}
H_{r} & =\frac{\mathrm{j}}{\omega \mu r} \frac{\partial E_{z}}{\partial \phi} \\
H_{\phi} & =-\frac{\mathrm{j}}{\omega \mu} \frac{\partial E_{z}}{\partial r} .
\end{aligned}
$$

Using these relations, the following field equations can be derived:

$$
H_{\phi}^{1}=-k\left[C_{1} J_{n}^{\prime}(k r)+C_{2} N_{n}^{\prime}(k r)\right] \cos (n \phi)
$$

where $J_{n}^{\prime}(k r)^{\prime}$ and $N_{n}^{\prime}(k r)$ are the first derivative of $J_{n}(k r)$ and $N_{n}(k r)$, respectively. Similarly,

$$
H_{r}^{1}=-\left(\frac{n}{r}\right)\left[C_{1} J_{n}^{\prime}(k r)+C_{2} N_{n}^{\prime}(k r)\right] \sin (n \phi) .
$$

In region II $(c \leqslant r \leqslant d)$, the solution of wave equation is given by

$$
E_{z}^{2}=-\mathrm{j} \omega \mu\left[C_{3} J_{n}(k r)+C_{4} N_{n}(k r)\right] \cos (n \phi) .
$$

Using the relation between fields, the following equations can be written as

$$
\begin{gathered}
H_{\phi}^{2}=-k\left[C_{3} J_{n}^{\prime}(k r)+C_{4} N_{n}^{\prime}(k r)\right] \cos (n \phi) \\
H_{r}^{2}=-\left(\frac{n}{r}\right)\left[C_{3} J_{n}^{\prime}(k r)+C_{4} N_{n}^{\prime}(k r)\right] \sin (n \phi)
\end{gathered}
$$

where $C_{3}$, and $C_{4}$ are the amplitude constants for region II. Considering the parasitic patch in isolation, the boundary condition of vanishing $H_{\phi}^{1}$ is applied, which yields the following expression:

$$
\int_{a}^{b}\left(-k\left[C_{1} J_{n}^{\prime}(k r)+C_{2} N_{n}^{\prime}(k r)\right] r \mathrm{~d} r\right)=0
$$

or

$$
\begin{gathered}
\int_{a}^{b}\left[C_{1} J_{n}^{\prime}(k r)+C_{2} N_{n}^{\prime}(k r)\right] r \mathrm{~d} r=0 \\
\int_{a}^{b} C_{1} J_{n}^{\prime}(k r) r \mathrm{~d} r+\int_{a}^{b} C_{2} N_{n}^{\prime}(k r) r \mathrm{~d} r=0 .
\end{gathered}
$$

Hence

$$
\frac{C_{2}}{C_{1}}=-\frac{\int_{a}^{b} J_{n}^{\prime}(k r) r \mathrm{~d} r}{\int_{a}^{b} N_{n}^{\prime}(k r) r \mathrm{~d} r}=\frac{I_{2}}{I_{1}} .
$$

Therefore, the field expression in region I can be rewritten as

$$
\begin{gathered}
E_{z}^{1}=-\mathrm{j} \omega \mu C_{n}^{1} F_{n}^{1}(k r) \cos (n \phi) \\
H_{\phi}^{1}=-k C_{n}^{1}{F_{n}^{1}}^{\prime}(k r) \cos (n \phi)
\end{gathered}
$$

where $C_{n}^{1}$ is a constant dependant on the mode $n$ and

$$
F_{n}^{1}(k r)=J_{n}(k r) I_{1}-N_{n}(k r) I_{2} .
$$

Using the boundary condition of vanishing the radial component of surface current at $r=d$, that is $H_{\phi}^{2}=0$ for $r=d$

$$
\frac{C_{3}}{C_{4}}=-\frac{N_{n}^{\prime}(k d)}{J_{n}^{\prime}(k d)}=\frac{I_{3}}{I_{4}}
$$

Thus the field expression in region II is

$$
E_{z}^{2}=-\mathrm{j} \omega \mu C_{n}^{2} F_{n}^{2}(k r) \cos (n \phi)
$$




$$
H_{\phi}^{2}=-k C_{n}^{2} F_{n}^{2^{\prime}}(k r) \cos (n \phi)
$$

where $C_{n}^{2}$ is a constant, and

$$
F_{n}^{2}(k r)=J_{n}(k r) I_{3}-N_{n}(k r) I_{4}
$$

Now, considering the gap between the two rings as a $\pi$-type network as shown in Fig. $3^{[1]}$. In Fig. $3, y_{n}^{w}(b)$ is the wall admittance of inner ring, $y_{n}^{w}(c)$ is the wall admittance of outer ring, and $y_{n}^{m}(b, c)$ is the mutual admittance between the two rings ${ }^{[18]}$. From the discontinuity of the current for gap in the structure:

$$
b H_{\phi}^{1}(b)-c H_{\phi}^{2}(c)=-E_{z}^{1}(b) Y_{1}-E_{z}^{2}(c) Y_{2}
$$

where $Y_{1}=y_{n}^{w}(b)+y_{n}^{m}(c, b)$, and $Y_{2}=y_{n}^{w}(c)-y_{n}^{m}(b, c)$. Using the field expressions in (22), the following expression can be obtained:

$$
\begin{aligned}
& -b k C_{n}^{1} F_{n}^{1^{\prime}}(k b)+c k C_{n}^{2} F_{n}^{2^{\prime}}(k c)= \\
& \quad \mathrm{j} \omega \mu C_{n}^{1} F_{n}^{1}(k b) Y_{1}+\mathrm{j} \omega \mu C_{n}^{2} F_{n}^{2}(k c) Y_{2}
\end{aligned}
$$

or

$$
\begin{array}{r}
C_{n}^{1}\left(-b k F_{n}^{1^{\prime}}(k b)-\mathrm{j} \omega \mu F_{n}^{1}(k b) Y_{1}\right)= \\
C_{n}^{2}\left(\mathrm{j} \omega \mu F_{n}^{2}(k c) Y_{2}-k c F_{n}^{2^{\prime}}(k c)\right)
\end{array}
$$

by rearranging

$$
\frac{C_{n}^{2}}{C_{n}^{1}}=\frac{\mathrm{j} \omega \mu F_{n}^{1}(k b) Y_{1}+b k F_{n}^{1^{\prime}}(k b)}{k c F_{n}^{2^{\prime}}(k c)-\mathrm{j} \omega \mu F_{n}^{2}(k c) Y_{2}}
$$

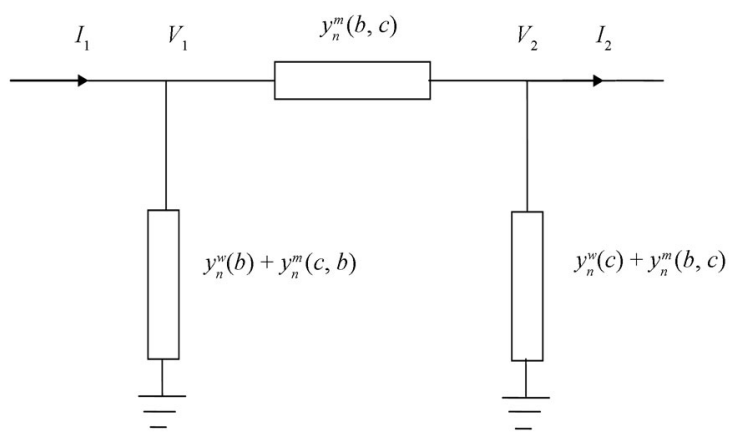

Fig. 3 Equivalent circuit diagram of the gap-coupled circular ring microstrip antenna

For small gap-distance between the rings, the tangential component of electric field is continuous at $r^{\prime}=0.5(b+c)$. The small gap is considered because for large gap the gap coupling becomes weaker, here maximum gap between the rings is taken as $0.15 d$. Therefore $E_{z}^{1}(r)=E_{z}^{2}(r)$ at $r=r^{\prime}$, applying it, following expression can be derived:

$$
\frac{C_{n}^{2}}{C_{n}^{1}}=\frac{F_{n}^{1}\left(k r^{\prime}\right)}{F_{n}^{2}\left(k r^{\prime}\right)}
$$

From (25) and (26), we get

$$
\frac{F_{n}^{1}\left(k r^{\prime}\right)}{F_{n}^{2}\left(k r^{\prime}\right)}-\frac{\mathrm{j} \omega \mu F_{n}^{1}(k b) Y_{1}+b k F_{n}^{1^{\prime}}(k b)}{k c F_{n}^{2^{\prime}}(k c)-\mathrm{j} \omega \mu F_{n}^{2}(k c) Y_{2}}=0
$$

Using (27), the resonant frequency of the structure is calculated.

\section{Results and discussion}

For simulation model, a schematic of feed ring and parasitic ring as shown in Fig. 1 is considered. The rings are placed on substrate of relative permittivity $\epsilon_{r}=2.2$ and thickness $h=1.59 \mathrm{~mm}$. The simulated results from computer simulation technology (CST) microwave studio and the analyzed results of the structure are depicted in Fig. 4 to Fig. 7. In Fig. 4, the variation of the resonant frequency of the structure with the inner radius of inner ring of gap-coupled circular ring microstrip antenna is shown. Here the radii $b, c$, and $d$ are taken constants with values $12.5 \mathrm{~mm}, 13 \mathrm{~mm}$, and $15 \mathrm{~mm}$, respectively. In Fig. 5 , the variation of resonant frequency with the outer radius of inner ring of gap-coupled circular ring microstrip antenna is shown. In Fig. 5 , the values of $a, c$, and $d$ are $7 \mathrm{~mm}, 13 \mathrm{~mm}$ and $15 \mathrm{~mm}$, respectively. In Fig. 6 , the variation of resonant frequency with the inner radius of outer ring of gapcoupled circular ring microstrip antenna is shown with the fixed values of $a, b$, and $d$ as $7 \mathrm{~mm}, 11.5 \mathrm{~mm}$, and $15 \mathrm{~mm}$, respectively. In Fig. 7 , the effect on resonant frequency due to variation in outer ring's outer radius is studied for the constant values of a, b and c as $7 \mathrm{~mm}, 11.5 \mathrm{~mm}$ and $12 \mathrm{~mm}$, respectively. From these figures it can be observed that the resonant frequency decreases on increasing the dimension of ring, and the analyzed results show good agreement with simulated results.

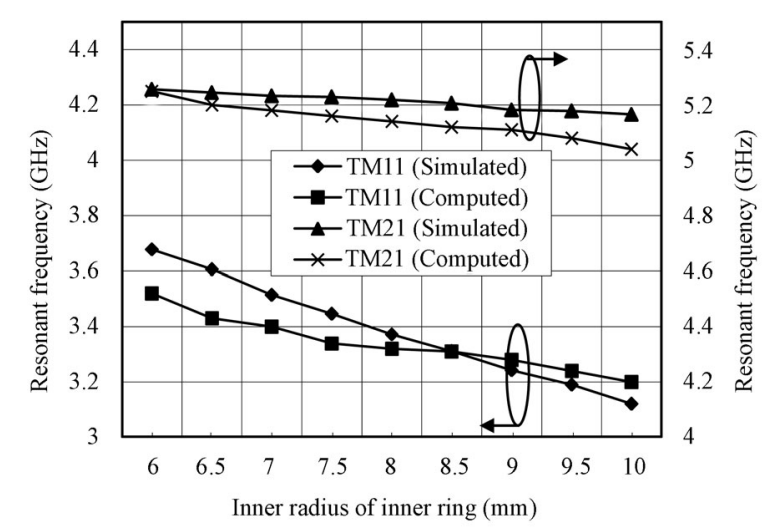

Fig. 4 Variation of resonant frequency with inner radius of the inner ring (outer radius of inner $\mathrm{ring}=12.5 \mathrm{~mm}$, inner radius of outer ring $=13 \mathrm{~mm}$, outer radius of outer ring $=15 \mathrm{~mm}$ ) 


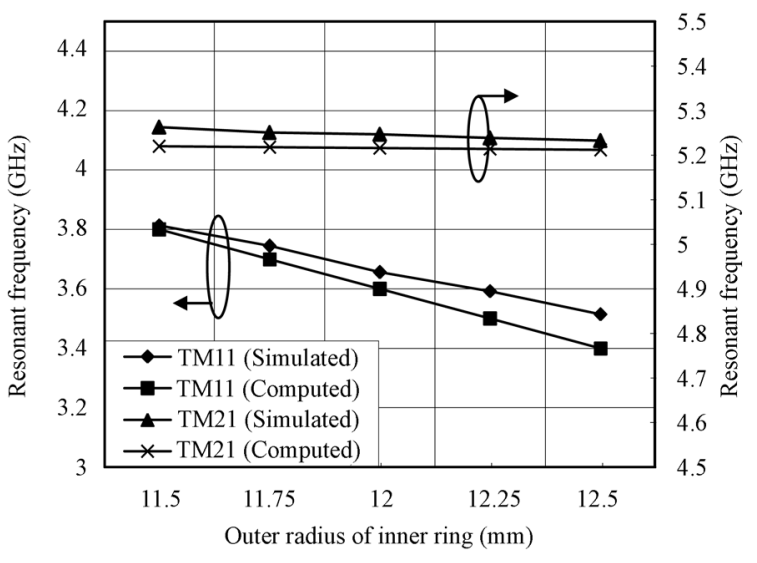

Fig. 5 Variation of resonant frequency with outer radius of inner ring (inner radius of inner ring $=7 \mathrm{~mm}$, inner radius of outer ring $=13 \mathrm{~mm}$, outer radius of outer ring $=15 \mathrm{~mm}$ )

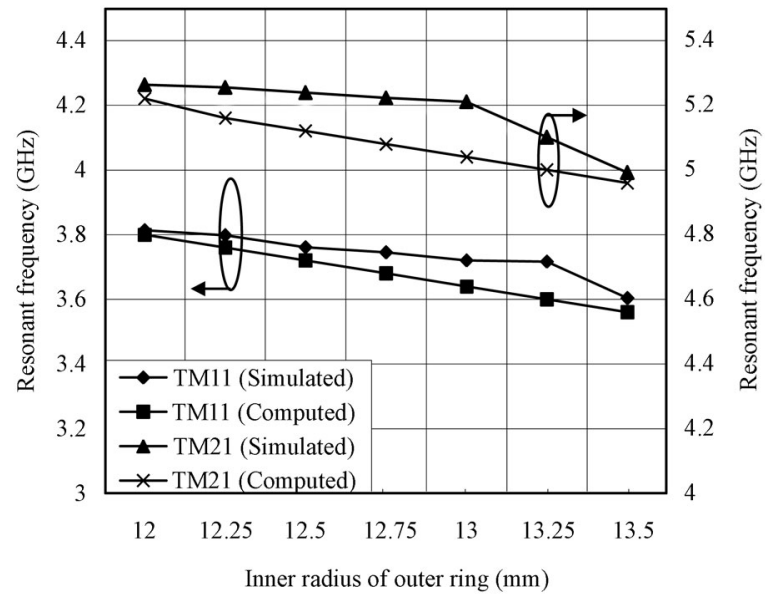

Fig. 6 Variation of resonant frequency with outer radius of inner ring (inner radius of inner ring $=7 \mathrm{~mm}$, outer radius of inner ring $=11.5 \mathrm{~mm}$, outer radius of outer ring $=15 \mathrm{~mm}$ )

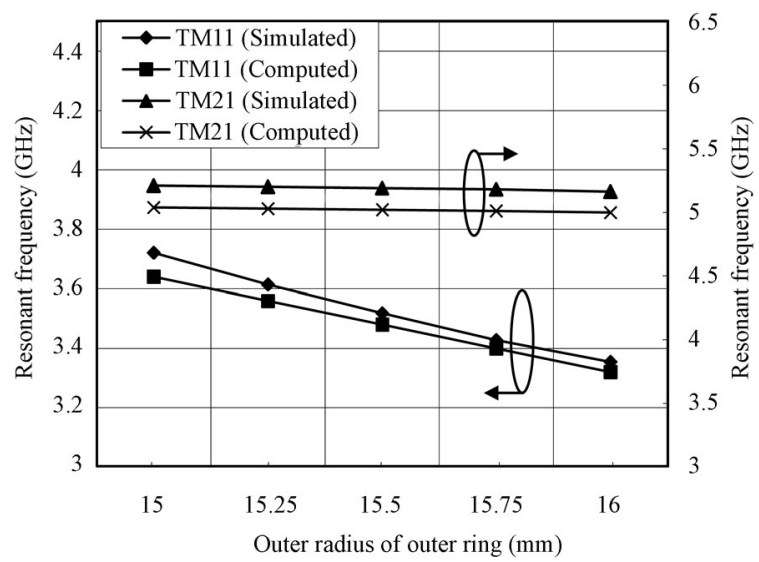

Fig. 7 Variation of resonant frequency with outer radius of outer ring (inner radius of inner ring $=7 \mathrm{~mm}$, outer radius of inner ring $=11.5 \mathrm{~mm}$, inner radius of outer ring $=12 \mathrm{~mm}$ )

\section{Conclusions}

In the present paper, an analytical model for computing the resonant frequency of gap-coupled circular microstrip ring antennas has been investigated. The comparison between the computed and simulated results shows good agreement in the resonant frequency. The two gap-coupled circular microstrip ring antennas can be used for wideband applications and for dual band applications. The proposed model can be extended to multiple ring resonators.

\section{References}

[1] R. Garg, P. Bhartia, I. Bahl, A. Ittipiboon. Microstrip Antenna Design Handbook, Boston: Artech House Publishers, 2001.

[2] C. A. Balanis. Antenna Theory: Analysis and Design, New Mexico: Wiley, 2005

[3] D. M. Pozer. Microstrip antennas. Proceedings of the IEEE, vol. 80, no 1, pp. 79-91, 1992.

[4] J. R. James, P. S. Hall. Handbook of Microstrip Antennas, London: Peter Prengrinus, 1989.

[5] A. K. Bhattacharya. Characteristics of circular patch on thick substrate and superstrate. IEEE Transactions on Antennas and Propagation, vol. 39, no. 7, pp. 1038-1041, 1991.

[6] M. P. Salmasi, F. H. Kashani, M. N. Azarmanesh. A novel broadband fractal sierpinski shaped, microstrip antenna. Progress in Electromagnetics Research C, vol. 4, pp.179190, 2008.

[7] J. H. Lu, C. L. Tang, K. L. Wong. Novel dual-frequency and broad-band designs of slot-loaded equilateral triangular microstrip antennas. IEEE Transactions on Antennas and Propagation, vol. 48, no. 7, pp. 1048-1054, 2000.

[8] K. L. Wong, J. Y. Jan. Broadband circular microstrip antenna with embedded reactive loading. Electronics Letters, vol. 34, no. 19, pp. 1804-1805, 1998.

[9] J. Y. Jan, K. L. Wong. Microstrip-line-fed broadband circular microstrip antenna with embedded reactive loading. Microwave and Optical Technology Letters, vol.22, no. 3, pp. 200-202, 1999.

[10] N. Fayyaz, S. Safavi-Naeini. Bandwidth enhancement of a rectangular patch antenna by integrated reactive loading. In Proceedings of IEEE Antennas and Propagation Society International Symposium Digest, IEEE, Atlanta, GA, USA, pp. 1100-1103, 1998.

[11] K. L. Wong, J. S. Kuo. Bandwidth enhancement of bowtie microstrip antennas using integrated reactive loading. Microwave and Optical Technology Letters, vol. 22, no. 1, pp. 69-71, 1999 . 
[12] F. Croq, G. Kossiavas, A. Papiernik. Stacked resonators for bandwidth enhancement: A comparison of two feeding techniques. IEE Proceedings $-H$ on Microwaves, Antennas and Propagation, vol. 140, no. 4, pp. 303-308, 1993.

[13] Z. Rasin, N. H. H. Khamis. Proximity coupled stacked configuration for wideband microstrip antenna. In Proceedings of the Asia-Pacific Conference on Applied Electromagnetics, IEEE, Melaka, pp. 1-4, 2007.

[14] C. K. Wu, K. L. Wong. Broadband microstrip antenna with directly coupled and parasitic patches. Microwave and $O p$ tical Technology Letters, vol. 22, no. 5, pp. 348-349, 1999.

[15] N. Ghassemi, J. Rashed-Mohassel, M. H. Neshati, M. Ghassemi. Slot coupled microstrip antenna for ultra wideband applications in $\mathrm{C}$ and $\mathrm{X}$ bands. Progress in Electromagnetics Research M, vol. 3, pp. 15-25, 2008.

[16] F. D. L. Peters, S. O. Tatu, T. A. Denidni. $77 \mathrm{GHz}$ microstrip antenna with gap coupled elements for impedance matching. Progress in Electromagnetics Research C, vol. 9, pp. 35-45, 2009.

[17] K. P. Ray, V. Sevani, A. A. Deshmukh. Compact gapcoupled microstrip antennas for broadband and dual frequency operations. International Journal of Microwave and Optical Technology, vol. 2, no. 3, pp. 193-202, 2007.

[18] P. Kumar, T. Chakravarty, G. Singh, S. Bhooshan, S. K. Khah, A. De. Numerical computation of resonant frequency of gap coupled circular microstrip antennas. Journal of Electromagnetic Waves and Applications, vol.21, no. 10 , pp. 1303-1311, 2007.
[19] P. Kumar, G. Singh. Gap-coupling: A potential method for enhancing the bandwidth of microstrip antennas. Advanced Computational Techniques in Electromagnetics, vol. 2012, Article ID acte-00110, 2012.

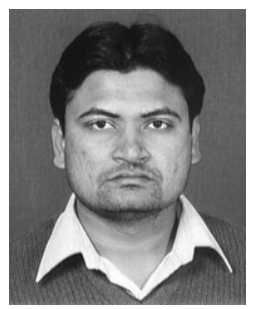

Pradeep Kumar graduated in electronics and communication engineering from Institute of Engineering and Technology, R U. Campus, India, and received his M. Eng. (Hons.) degree in electronics and communication engineering from Thapar Institute of Engineering and Technology (Deemed University) (now Thapar University), India, and his Ph. D. degree in electronics and communication engineering from Jaypee University of Information Technology, India. He completed his postdoctoral studies from Autonoma University of Madrid, Spain. He was associated with Mody Institute of Technology and Science (Deemed University) Lakshmangarh, India.

$\mathrm{He}$ is the author of many research papers published in various journals and conferences. He is serving as reviewer/member of editorial board/technical editor/associte editor to many journals. He served as member of technical programme committee/chair/coordinator for many international conferences. $\mathrm{He}$ has received many fellowships/awards. He is the life member of The Institution of Electronics and Telecommunications Engineers, India. He is member/senior member of many professional studies such as International Association of Computer Science and Information Technology, Rajasthan Physics Association (RPA), Science Advisory Board, etc. Presently, he is working as associate professor with Jaypee University of Information Technology, India.

His research interests includes antenna and array design, signal processing, and image processing.

E-mail: pkumar-123@yahoo.com 